\title{
Early Mobility Following Hip Fracture Surgery with Furlong Bipolar Hemiarthroplasty-Cemented Versus Hydroxyapatite Coated Uncemented Prostheses
}

\author{
Shah Jehan ${ }^{1 *}$, Marek Parkola ${ }^{2}$, Javed Salim ${ }^{3}$, Susan Wilcockson ${ }^{4}$ and Thomas Foulstone ${ }^{5}$ \\ ${ }^{1}$ Specialty Trainee Trauma and Orthopaedic Surgery, Hull Royal Infirmary, UK \\ ${ }^{2}$ Foundation Year 2 Doctor, Trauma and Orthopaedic Surgery \\ ${ }^{3}$ Consultant Trauma and Orthopaedic Surgery \\ ${ }^{4}$ Senior Clinical Specialist Physiotherapist for Orthopaedics \\ ${ }^{5}$ Physiotherapist for Orthopaedics
}

Received: January 08, 2018; Published: January 18, 2018

*Corresponding author: Shah Jehan, Specialty Trainee Trauma and Orthopaedic Surgery, Hull Royal Infirmary, Hull, UK, HU3 2JZ, Tel: +447898984139; Email: shahjehan200@gmail.com

\begin{abstract}
Mobility is an important factor in the rehabilitation phase for hip fracture patients. Among many factors affecting postoperative mobility, stability of the stem following hemiarthroplasty is considered crucial for early mobility. The purpose of this study was to compare early postoperative mobility in patients with cemented and uncemented hydroxyapatite coated Furlong hemiarthroplasty. We performed a retrospective comparative study to compare two groups of patients who had either cemented, or hydroxyapatite (HA) coated uncemented implants over a period of six months. The groups were matched for their age, sex, pre-fracture mobility and accommodation prior to injury. Their early post-operative (day1-day7) mobility was assessed based on their walking distance, need for support, and pain level. The length of hospital stay and discharge destinations were also recorded and compared between the groups. Each group had 22 patients. The average age was 84 years (range, 74-95). The average pre-fracture mobility score was 5.5. The average post-operative mobility for cemented group was 2.7 at day 1 and 6.3 at day 7. For the uncemented group the average post-operative mobility at day 1 was 3.7 , and at day 7 it was 6.9 . Hospital stay was slightly longer in the cemented group. We conclude that early post-operative mobility is slightly better in patients who had HA coated uncemented implants. The average hospital stay is longer in the group with cemented implants.
\end{abstract}

Keywords: Hemiarthroplasty; Hip; Fracture; Mobility; Orthopaedic; Trauma; Rehabilitation

Abbreviations: NICE: National Institute of Clinical Excellence; HA: Hydroxy Apatite; OH: Own Home; RH: Residential Homes

\section{Introduction}

Early discharge from hospital should be the aim of the treatment in patients with neck of femur fractures [1]. There are many causes of prolonged hospital stay in these patients; poor mobility is one of the major factors. Patients with poor mobility also need extended support at discharge. The National Institute of Clinical Excellence (NICE) suggests that all the patients with neck of femur fractures should be treated with an aim to mobilise without any restriction [2]. Poor early post-operative mobility is related to a high rate of inpatient complications and prolonged rehabilitation. For the displaced intracapsular neck of femur fractures, the most common method of treatment is hemiarthroplasty [3]. There has been a recent increase in the use of cemented hemiarthroplasty due to reported better post-operative mobility and pain reduction $[4,5]$. However in these studies, the outcome has been analysed in relation to cementing of the prosthesis only. Other design features and hydroxyapatite coating of the prostheses have not been taken into account. These factors can affect the stability and hence the outcome in form of mobility and pain. When hydroxyapatite coated prostheses are compared with cemented prostheses, functional outcome may be comparable [6]. We performed a retrospective comparative study to compare the results of cemented and uncemented HA coated bipolar Furlong prostheses. The outcome measures were early mobility, length of hospital stay, and requirement of support at discharge. 


\section{Patients and Methods}

We performed a retrospective comparative study to compare the early post-operative mobility of patients who had either cemented or uncemented Furlong bipolar hemiarthroplasty (JRI Orthopaedics, Sheffield S35 2PY, UK). Data were collected for all patients going for hemiarthroplasty over a period of 6 months. The patients were then divided into two groups. Group 1 had hydroxyapatite (HA) coated uncemented stem and group 2 had cemented stem (Figure 1). Bipolar heads were used in both groups. The mobility levels prior to the fracture were recorded using the mobility scoring system proposed by Parker et al. [7] Table 1 shows the pre-fracture mobility scoring system. In our institute, the trend has been shifting from uncemented to cemented prostheses as suggested by NICE guidelines [2].

Table 1: Preoperative mobility scores (0-9).

\begin{tabular}{|c|c|c|c|}
\hline Mobility & No Difficulty & With an aid & With help from another person \\
\hline Able to get about the house & 3 & 2 & 0 \\
\hline Able to get out of the house & 3 & 2 & 1 \\
\hline Able to go shopping & 3 & 2 & 1 \\
\hline
\end{tabular}

Therefore, there were more patients with cemented prostheses compared to uncemented prostheses. We identified 22 patients who had uncemented hemiarthroplasty over a period of six months. We then matched 22 patients from cemented group to compare their early post-operative mobility. The patients were matched for their pre-fracture mobility level, age, sex and pre-fracture

accommodation. We defined early mobility as mobility for the first week in the post-operative period. The mobility was assessed and recorded for the first seven days, taking first post-operative day as day 1 . For each patient, the mobility score was recorded from 0-9 depending on the accumulative score of three variables (Table 2).

Table 2: Mobility assessment scoring in the early post-operative period (0-9). Three variables were used to score mobility.

\begin{tabular}{|c|c|c|c|c|}
\hline \multirow{2}{*}{ Walking distance } & Not mobilising & Bed to chair & Less than 5 steps & More than 5 steps \\
\cline { 2 - 5 } & 0 & 1 & 2 & 3 \\
\hline \multirow{2}{*}{ Support Requirement } & Not mobilising & With human help & With aid & 3 \\
\cline { 2 - 5 } & 0 & 1 & 2 & Moderate pain \\
\hline \multirow{2}{*}{ Pain during mobilisation } & Not mobilising & Severe pain & 2 & 3 \\
\cline { 2 - 5 } & 0 & 1 & Mild or No pain \\
\hline
\end{tabular}

The scoring system for post-operative mobility is different from that used to record the pre-operative mobility. This is because the pre-operative scoring system incorporates outdoor mobility which was not applicable in early post-operative period. Therefore, it was not possible to compare the pre and post-operative mobility in the same group. However, pre-fracture mobility scores helped us to match the groups for their pre-operative mobility. The information about the nature of accommodation prior to injury was collected at admission. We also collected data for type of anaesthesia, length of stay and discharge destination. We calculated average mobility scores from day 1 to day 7 . The scores were then compared using unpaired t-test to see if there was any difference between the two groups.

\section{Results}

There were a total of 44 patients with 22 patients in each group. Table 3 shows the demographic data for patients. The patients were matched for their age, sex, and pre-fracture accommodation. The average age was 84 years. There were 15 female and 7 male patients in uncemented group and 14 females and 8 males in cemented group. In each group, 16 patients (72.7\%) were admitted from their own home $(\mathrm{OH})$, and 6 patients admitted from residential homes $(\mathrm{RH})$. The average pre-fracture mobility was 5.5 in both groups.

Table 3: Demographic data for cemented and un-cemented groups $\mathrm{OH}=$ own home, $\mathrm{RH}=$ residential home. Both the groups were matched for age, sex, pre-fracture mobility and pre-fracture accommodation.

\begin{tabular}{|c|c|c|}
\hline & Cemented (22) & Uncemented (22) \\
\hline Average age (range) & $84(72-95)$ & $84(74-95)$ \\
\hline Male : Female & $8: 14$ & $7: 15$ \\
\hline Admission from (OH:RH) & $16: 6$ & $15: 7$ \\
\hline $\begin{array}{c}\text { Average pre-fracture } \\
\text { mobility score (range) }\end{array}$ & $5.5(2-8)$ & $5.5(2-9)$ \\
\hline
\end{tabular}

In the cemented group, 15 patients had general anaesthesia and 7 had spinal anaesthesia; while in the uncemented group 16 had general anaesthesia and 6 had spinal. The average delay from admission to operation was 1.5 days (range, 1-5) for the 
uncemented group, and 1.7 days (range, 1-6) for the cemented group. The post-operative mobility scores are shown in Figure 2. The average mobility score for the uncemented group at day 1 was 3.7 and for the cemented group it was 2.7. Both the groups showed steady improvement in mobility from day-1 (D1) today-7(D7). At day 7 the average mobility score for uncemented group was 6.9 and for cemented group it was 6.3. Although the average mobility for the uncemented group at D7 was slightly better than the cemented group, there was no significant difference between the two groups $(\mathrm{p}=0.32)$.

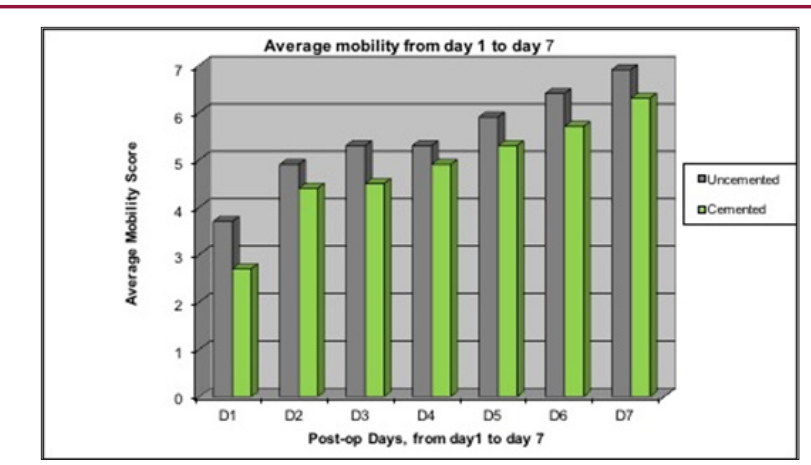

Figure 2: Early post-op mobility average scores, comparison between cemented and uncemented cases.

The average hospital stay for cemented group was and 15 days (range, 4-24) and for uncemented group it was 13 days (range,4-23). One patient from each group died in hospital, therefore their length of stay was excluded while calculating length of hospital stay. Figure 3 shows the destination at discharge. From the uncemented group 10 patients $(45.5 \%)$ were discharged to their own home, from the cemented group 8 patients (36.3\%) were discharged to their own home. Nine patients from the cemented group and 8 from the uncemented group were discharged to residential homes. Two patients from the uncemented group and one from the cemented group needed intermediate care team assistance at the time of discharge to their own home. Two patients from each group were discharged to community hospitals for further rehabilitation.

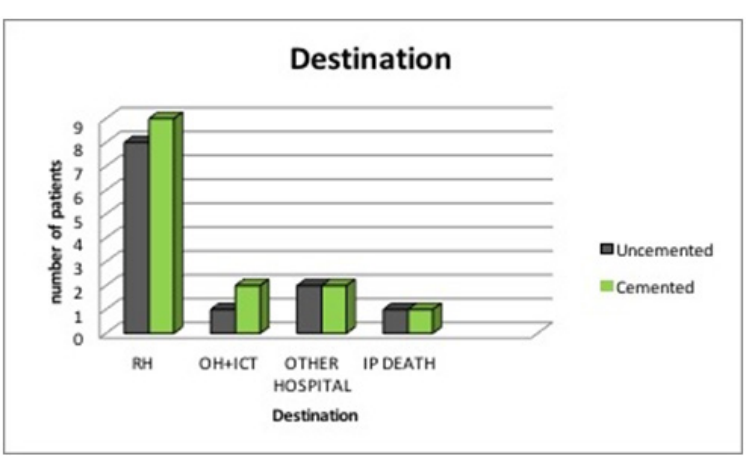

Figure 3: Destination at discharge, one patient from each group died during hospital stay. $\mathrm{OH}=$ own home, $\mathrm{RH}=$ residential home, $\mathrm{OH}+\mathrm{ICT}=$ own home with help from intermediate care team, $\mathrm{IP}=$ inpatient.

\section{Discussion}

Hip fractures are related to high morbidity and mortality especially in the elderly population [8]. Mortality in the first year can reach up to $20 \%$, and the majority of the people who recover from hip fractures need additional support for routine activities $[9,10]$. Poor mobility is an important cause not only for the initial fall and resultant hip fracture in the first instance, but also for the prolonged rehabilitation and extended support at discharge following surgery. Loss of independence because of poor mobility has a major impact on quality of life in the elderly population, and most these people fail to achieve their pre-fracture functional status [11,12]. Early post-operative mobilisation is considered an important factor for prevention of complications related to prolonged decumbency as well as for effective rehabilitation [13].

Poor post-operative physical function is related to a high rate of institionalization compared to patients who have high physical function [14]. The National Institute of Clinical Excellence (NICE) therefore suggests that patients should be operated with the aim to mobilise without any restriction, and mobilisation should be offered by physiotherapist on the first day after surgery [2]. Hemiarthroplasty is the mainstay of treatment for intracapsular neck of femur fractures. Mobility is thought to be better if the stem is stable in the femoral canal. In this regard, cemented implants were considered to provide good stability, especially in old patients with osteoporotic bones [15]. However, osteoporosis and poor bone quality cannot be regarded as contraindications to HA coated implants, and good stability is achievable if the initial filling of the femoral canal is satisfactory $[15,16,17]$. Therefore, the femoral canal preparation is an important step for better filling with the stem and over reaming should be avoided if using an uncemented implant [15]. Previous literature suggests that cemented implants result in better mobility compared to the uncemented implants $[4,5]$.

However, in these studies, diversity of the implants can be a cofounding factor. In addition, in the majority of these studies cemented implants were compared to uncoated uncemented implants. When hydroxyapatite coated uncemented implants were compared to cemented implants no difference in outcome was noted at 3 months, or at one year follow up. The duration of surgery and blood loss was less in the uncemented group [6]. The authors concluded that both cemented and HA coated implant can be used with good results in displaced neck of femur fractures [6]. In their case series, Chandran $P$ et al reported the results 112 patients of HA coated Furlong hemiarthroplasty at average 4 year follow up [18]. They concluded good results in terms of mobility and freedom from pain.

In the early post-operative period, mobility is not dependent on a single factor. Patient's age, pre-fracture mobility, type of anaesthesia and pain level is all important and should be taken into consideration. In our study, the patients were matched for age, sex and pre-fracture mobility level. Our post-operative scoring system for mobility was based on walking distance (0-3), support requirement (0-3) pain during mobilisation (0-3). The scores from these three variables were then added to achieve the cumulative score. The scoring system was discussed extensively with our senior physiotherapist and geriatrician and thought to be reflective of early post-operative mobility. We think this scoring system can be used for early mobility assessment in hip fracture patients for future studies. 


\section{Conclusion}

Our study only focused on early mobility and hospital stay and we did not observe any benefit with cemented implant. The group with uncemented implants showed better mobility scores and comparatively early discharge from hospital although this was not significant. The strengths of study include; well matched groups (age, sex and pre-fracture mobility) and independent collection of data by physiotherapists. The implants used in both groups had bipolar heads (Furlong bipolar systems). The only difference was in stems i.e. uncemented HA coated stem versus cemented stem. There are some weaknesses of the study as well. A single surgeon did not perform the surgery. Therefore, the effect of surgical technique can influence the mobility. However, in all the patients the modified Hardinge approach was used. The study was limited only to mobility while the patients were in hospital.

\section{References}

1. Sikorski JM, Davis NJ, Senior J (1985) the rapid transit system for patients with fractures of proximal femur. British medical J (Clin research ed) 290: 439-443.

2. (2011) the management of hip fracture in adults, CG124 NICE Clinical guidelines. National Institute for Health and Clinical Excellence mid City Place 71 High Holborn, Londo, UK.

3. Leighton RK, Schmidt AH, Collier P, Trask K (2007) Advances in the treatment of intracapsular hip fractures in the elderly. Injury 38: 24-34.

4. Parker MI, Pryor G, Gurusamy K (2010) Cemented versus uncemented hemiarthroplasty for intracapsular hip fractures: A randomised controlled trial in 400 patients. J Bone Joint Surg Br 92(1): 116-122.

5. Parker MJ, Gurusamy KS, Azegami S (2010) Arthroplasties (with and without bone cement) for proximal femoral fractures in adults. Cochrane database of systematic reviews.

6. Figved W, Opland V, Frihagen F, Jervidalo T, Madsen JE, et al. (2009) Cemented versus uncemented hemiarthroplasty for displaced femoral neck fractures. Clinical orthopaedics and related research 467(2): 2426 2435.

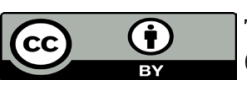

This work is licensed under Creative Commons Attribution 4.0 License

Submission Link: http://biomedres.us/submit-manuscript.php
7. Parker MJ, Palmer CR (1993) A new mobility score for predicting mortality after hip fracture. J Bone Joint Surg Br 75(5): 797-798.

8. Kannus P, Niemi S, Palvanen M, Parkkari J (1997) Fall-induced injuries among elderly people. Lancet 350: 1174.

9. Cumming RG, Nevitt MC, Cummings SR (1997) Epidemiology of hip fractures. Epidemiologic reviews 19(2): 244-257.

10. Mossey JM, Mutran E, Knott K, Craik R (1989) Determinants of recovery 12 months after hip fracture: the importance of psychosocial factors. Am J public health 79(3): 279-286.

11. Salkeld G, Cameron ID, Cumming RG, Easter S, Seymour J, et al. (2000) Quality of life related to fear of falling and hip fracture in older women: a time trade off study. BMJ 320:341-346.

12. Murphy S, Conway C, McGrath NB, OLeary B, OSullivan MP, et al. (2011) An intervention study exploring the effects of providing older adult hip fracture patients with an information booklet in the early postoperative period. J Clin Nursing 20(23-24): 3404-3413.

13. Handoll HHG, Sherrington C (2017) Mobilisation strategies after hip fracture surgery in adults. Cochrane database of systematic reviews.

14. Cree M, Soskolne CL, Belseck E, Hornig J, McElhaney JE, et al. (2000) Mortality and institutionalization following hip fracture. J the Am Geriatrics Soc 48(3): 283-288.

15. Oztürkmen Y, Karamehmetoğlu M, Caniklioğlu M, Ince Y, Azboy I (2008) Cementless hemiarthroplasty for femoral neck fractures in elderly patients. Indian J orthopaedics 42(1): 56-60.

16. McAuley JP, Moore KD, Culpepper WJ, Engh CA (1998) Total hip arthroplasty with porous-coated prostheses fixed without cement in patients who are sixty-five years of age or older. The J of bone and joint surgery Am 80(11): 1648-1655.

17. Cracchiolo A, Severt R, Moreland J (1992) Uncemented total hip arthroplasty in rheumatoid arthritis diseases. A two- to six-year followup study. Clinical orthopaedics and related research 277: 166-174.

18. Chandran P, Azzabi M, Burton DJC, Andrews M, Bradley JG (2006) Midterm results of Furlong LOL uncemented hip hemiarthroplasty for fractures of the femoral neck. Acta orthopaedica Belgica 72(4): 428-433.

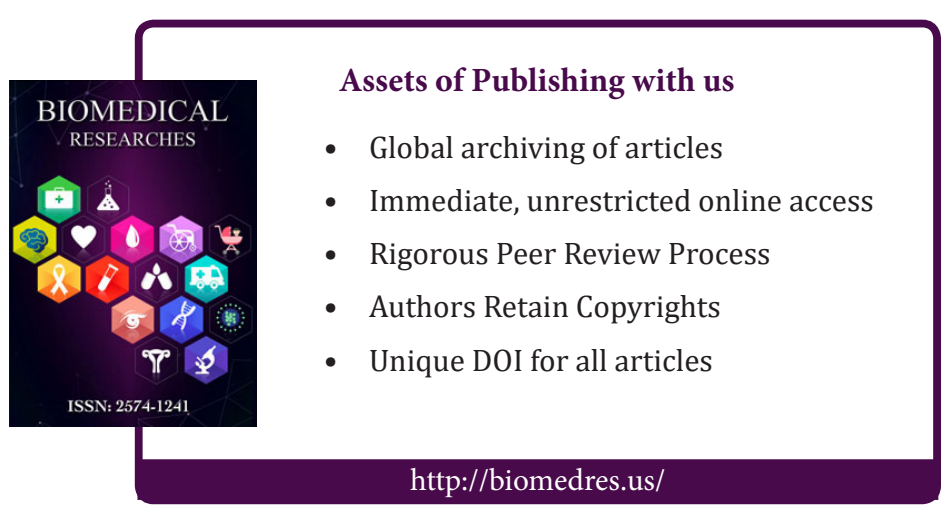

